\title{
Analysis and Research on Flow Characteristics of Hot Water Pipeline System
}

\author{
Hou Yingzhe ${ }^{1}$, Wu Hao ${ }^{1}$, Yan Xiaozhe ${ }^{1}$, Zhu Haoqiang ${ }^{1}$, Gao Haitao ${ }^{1}$, Hu Yiming ${ }^{1}$, Cui Qiguan ${ }^{1}$, Xiong Jinying ${ }^{1}$, \\ Zhou Longyu ${ }^{1}$ \\ ${ }^{1}$ Wuhan Secondary Ship Design and Research Institute, Wuhan, Hubei, 430205, China
}

\begin{abstract}
Based on the CFD numerical simulation method, a pipe model was established to study the internal flow characteristics of two different specifications of hot water pipe system, and the related information of the flow field was obtained, such as the internal pressure, flow velocity and flow rate. The results showed that the smoother the pipeline transition was, the smaller the velocity uniformity coefficient would be, and the higher flow field uniformity means the smaller pressure and velocity fluctuations. Therefore, the pipeline vibration will be smaller, and the flow characteristics are greatly improved.
\end{abstract}

\section{INTRODUCTION}

Hot water pipeline system is widely used in energy, shipping, petrochemical, machinery and other industries. As for the power system, it serves as the bridge and link to connect the boiler, heat accumulator, heat exchanger and other equipment. The unreasonable pipeline design and layout will lead to the loss of the pipeline resistance. Too large resistance fails to ensure the smooth medium flow, while too small resistance will make the medium flow too large. And the large flow rate will bring pipeline vibration, affecting the safety and reliability of the system. At the same time, in the large-scale pipe network, the number of pipelines is large, add to the various specifications, and the complex layout. The layout and direction of the pipe network have great limitations due to the limited space. Therefore, the design of the pipe network should not only meet the requirements of completing the pipeline system layout in the limited space, but also ensure that all equipment parameters can meet the requirements of stable operation.

In this paper, we do research on the flow characteristics of the valve test system, which is designed for the operation performance test and discharge test of the pressurizer safety valve. We analyze two different pipe combinations to improve the flow characteristics, and propose the optimization scheme of pipe system combination.

\section{STRUCTURE PRINCIPLE PERFORMANCE PARAMETERS}

\subsection{Structure principle}

AND
The hot water test circuit is mainly composed of heat (19 $\mathrm{m}^{3}$ ) accumulator, hot water container $\left(5 \mathrm{~m}^{3}\right)$, valve, pipeline and accessories, as shown in Figure 1.

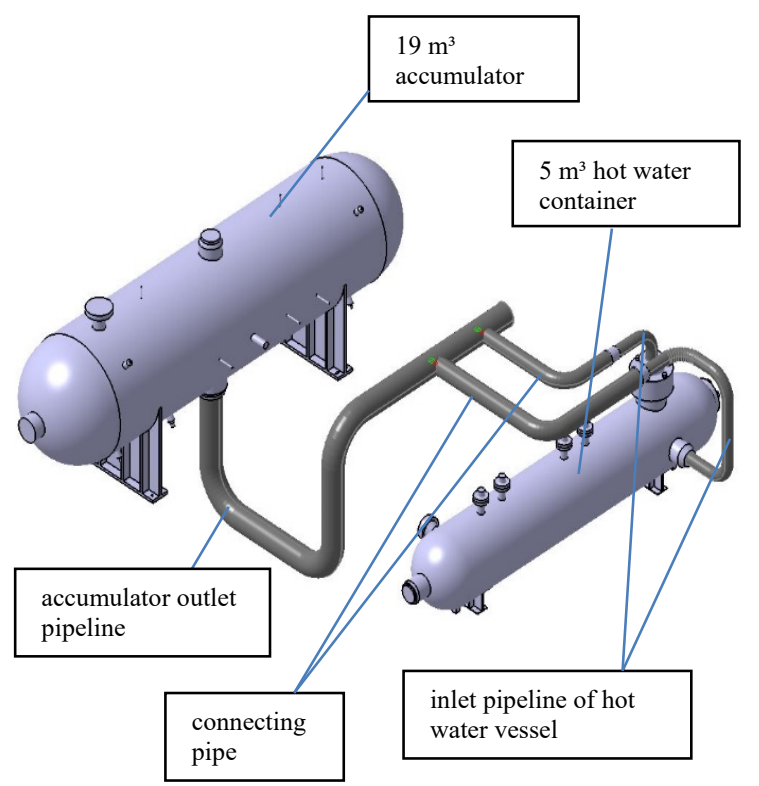

1. $19 \mathrm{~m}^{3}$ accumulator

2. $5 \mathrm{~m}^{3}$ hot water container

3. accumulator outlet pipeline

4. connecting pipe

5. inlet pipeline of hot water vessel

Figure 1. Structure diagram of hot water pipeline system

\subsection{Performance Parameters}

Parameters of Hot Water Pipeline System (Table 1):

Table 1. Parameters of hot water pipeline

\begin{tabular}{|c|c|}
\hline Name & Numerical value \\
\hline accumulator volume & $19 \mathrm{~m}^{3}$ \\
volume of hot water container & $5 \mathrm{~m}^{3}$ \\
\hline
\end{tabular}




\begin{tabular}{|c|c|}
\hline maximum test pressure $(\mathrm{MPa})$ & 20 \\
\hline temperature range $\left({ }^{\circ} \mathrm{C}\right)$ & $333 \sim 343$ \\
\hline medium & $\begin{array}{c}\text { Saturated water } / \\
\text { super cooled water }\end{array}$ \\
\hline accumulator outlet pipeline & $\varphi 406 \times 25$ \\
connecting line & $\varphi 273 \times 34 / \varphi 325 \times$ \\
inlet pipeline of hot water vessel & 40 \\
\hline
\end{tabular}

This paper compares and analyzes the two connecting pipe specifications from $19 \mathrm{~m}^{3}$ heat storage device to $5 \mathrm{~m}^{3}$ water vessel pipeline system. The specific system pipeline combination is as follows:

(1)Plan A:

Accumulator outlet pipeline $\varphi 406 \times 25$

Connecting line $\varphi 273 \times 34$

Inlet pipeline of hot water vessel $\varphi 273 \times 34$

(2)Plan B:

Accumulator outlet pipeline $\varphi 406 \times 25$

Connecting line $\varphi 325 \times 40$

Inlet pipeline of hot water vessel $\varphi 273 \times 34$

\section{Numerical Simulation}

\subsection{Governing equation}

In this paper, the mature standard model is used for numerical simulation. The continuity equation, momentum conservation equation, turbulent kinetic energy $\mathrm{k}$ equation and dissipative energy equation constitute the governing equations.

Continuity equation:

$$
\frac{\partial \rho}{\partial t}+\frac{\partial}{\partial x_{i}}\left(\rho u_{i}\right)=0
$$

Momentum conservation equation (N-S equation) formula:

$$
\begin{aligned}
& \rho \frac{d u}{d t}=\frac{\partial p}{\partial x_{i}}+\frac{\partial}{\partial x_{j}}\left[\mu\left(\frac{\partial u_{i}}{\partial x_{j}}+\frac{\partial u_{j}}{\partial x_{i}}-\frac{2}{3} \delta_{i j} \frac{\partial u_{i}}{\partial x_{j}}\right)\right] \\
& +\frac{\partial}{\partial x_{j}}\left(-\overline{u_{i}^{\prime} u_{j}^{\prime}}\right)
\end{aligned}
$$

The transport equations of turbulent kinetic energy $\mathrm{k}$ and turbulent energy dissipation rate of standard $k \sim \varepsilon$ turbulence model can be expressed as follows:

$$
\begin{aligned}
& \frac{\partial(\rho k)}{\partial t}+\frac{\left(\partial \rho k u_{i}\right)}{\partial x_{i}}=\frac{\partial}{\partial x_{i}}\left[\left(\mu+\frac{\mu_{t}}{\delta_{k}}\right) \frac{\partial k}{\partial x_{j}}\right]+ \\
& P-\rho \varepsilon \\
& \frac{(\partial \rho \varepsilon)}{\partial t}+\frac{\left(\partial \rho \varepsilon u_{i}\right)}{\partial x_{i}}=\frac{\partial}{\partial x_{j}} \mu+\frac{\mu_{t}}{\sigma_{Z}} \frac{\partial \varepsilon}{\partial x_{j}} \\
& +\frac{\varepsilon}{k}\left(C_{\varepsilon 1} P+\rho C_{\varepsilon 2} \varepsilon\right)
\end{aligned}
$$

In the formula

$u_{i}$ _Velocity component along direction $\mathrm{i}, \mathrm{i}=1$,

2,3

$\mu$ _ Kinematic viscosity coefficient of medium

$u_{t}$ _ Eddy viscosity coefficient

$\delta_{k}$ _ Prandtl number corresponding to turbulent kinetic energy $\mathrm{k}$

$\delta_{\varepsilon}$ _ Prandtl number corresponding to turbulent dissipation rate $\mathcal{E}$

$\mathrm{P}$ - Generation term of turbulent kinetic energy

$$
P=\mu_{t}\left(\frac{\partial u_{i}}{\partial x_{j}}+\frac{\partial u_{j}}{\partial x_{i}}\right) \frac{\partial u_{i}}{\partial x_{j}}
$$

where:

$$
\mu_{t}=C_{\mu} \rho \frac{k^{2}}{\varepsilon}
$$

Empirical constants in model k- $\varepsilon$ (Table 2).

Table 2. Coefficients in $k-\varepsilon$ model

\begin{tabular}{ccccc}
\hline$C_{\varepsilon 1}$ & $C_{\varepsilon 2}$ & $C_{\mu}$ & $\delta_{k}$ & $\delta_{\varepsilon}$ \\
\hline 1.44 & 1.92 & 0.09 & 1.0 & 1.3
\end{tabular}

\subsection{Establishment of flow channel model and grid division}

According to the specific structural parameters, CATIA software is used to establish the three-dimensional model of the pipeline system, and the internal flow path model is generated by introducing the reverse modeling of the hydraulic software. The flow model is introduced into ANSYS ICEM CFD for mesh division (Figure 2). The numerical calculation grid is divided by tetrahedron or hybrid grid. We generate more accurate calculation results through the local encryption at the corner of convection channel, and then perform the grid independence test.

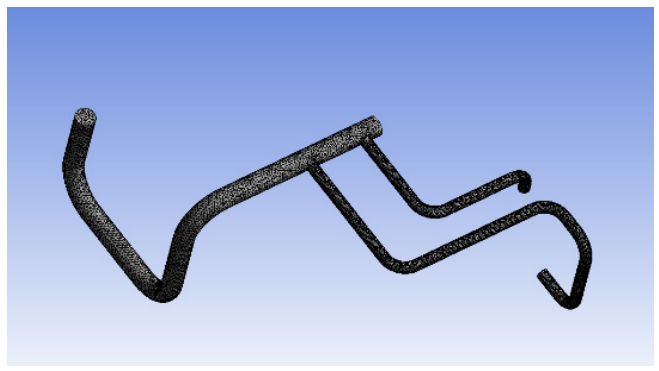

a) Plan $A$ 


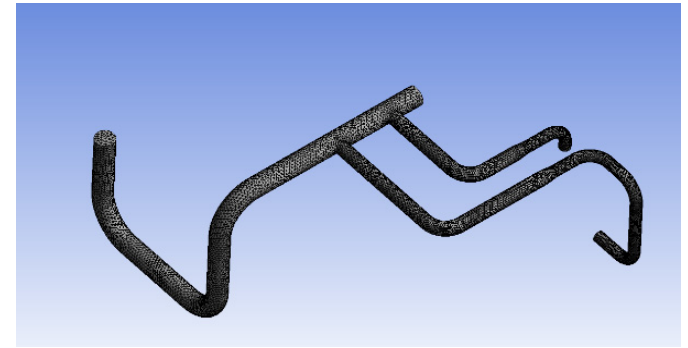

b) Plan B

Figure 2. Grid model of pipeline system

Combined with the actual working conditions of hot water pipeline system, the internal flow model under typical conditions is established. The closed equations are composed of three-dimensional incompressible Reynolds time average equations and $\mathrm{k}-\varepsilon$ turbulence model to solve the internal flow field of the pipeline system. The fluid medium is water, and the temperature change is not considered in the solution process. The outlet of the heat accumulator is set as the boundary condition of the velocity inlet, the velocity is $6.484 \mathrm{~m} / \mathrm{s}$, the inlet 1 and 2 of the hot water vessel are set as the boundary conditions of the pressure outlet of $0 \mathrm{MPa}$, and the pipeline wall should be set as the adiabatic and slip free boundary condition, then we can simulate and analyze the three-dimensional flow field.

\section{Study on Internal Flow Characteristics}

\subsection{Pressure Field Distribution}

In Figure 3, it shows the combined pressure cloud diagram of different pipe specifications. The figures indicate that the pressure gradient of Plan A changes greatly with uneven pressure distribution, and the maximum pressure appears at the end of the pipeline. While the pressure gradient of Plan B changes little, its pressure fluctuation has a smooth transition and the distribution is relatively uniform.
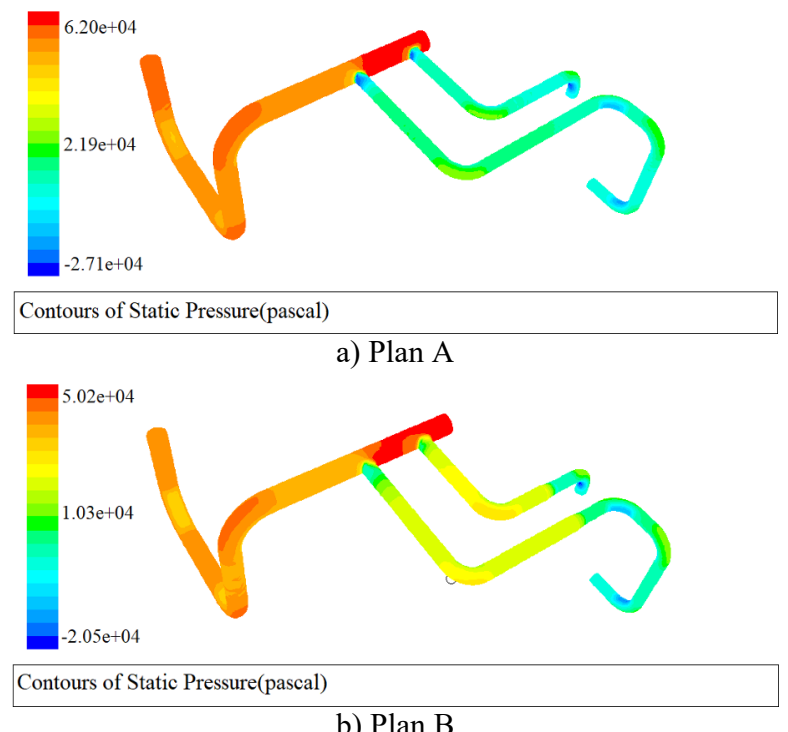

Figure 3. Pressure nephogram of different pipeline specifications

\subsection{Velocity field distribution}

After studying the velocity field distribution of different pipe combinations, we have obtained the velocity distribution nephogram, as shown in Figure 4. According to the figures, the velocity of Plan A is high and the velocity gradient changes greatly. The maximum velocity appears at the junction between the outlet pipe of the heat exchanger and the connecting pipe, causing certain scour to the pipeline. We can see gradual changes in Plan B, and the velocity distribution is uniform and tends to be stable.

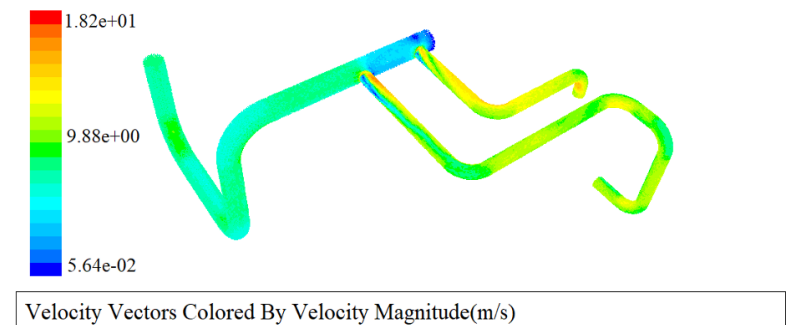

Velocity Vectors Colored By Velocity Magnitude(m/s)

a) Plan $\mathrm{A}$

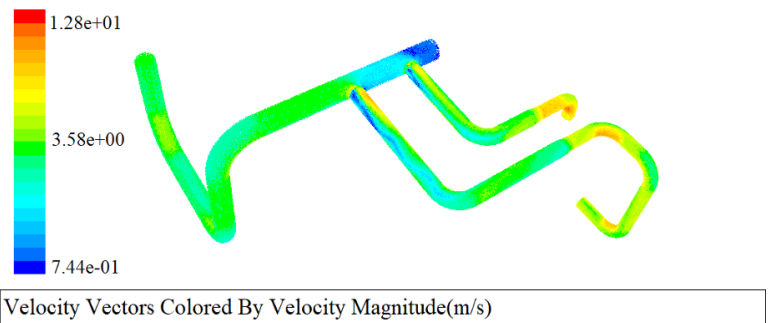

b) Plan B

Figure 4. Velocity nephogram of different pipeline specifications

\section{Conclusion}

(1) Compared with Plan A, Plan B is more optimized by using the $\varphi 325 \times 40$ connecting pipeline, and the change of pressure gradient and velocity gradient is more stable.

(2) The more stable the pipeline combination transition, the higher the uniformity of flow field, the smaller the pressure and velocity fluctuations, and the smaller the pipeline vibration, the flow characteristics will be greatly improved.

\section{References}

1. Wang Huihui.Study on flow characteristics and noise of pipeline system [D]. Huazhong University of Science and Technology, 2017. 5.

2. Zhang Zhiyong, Shen Rongying. Analysis of the vibration response of the liquid filled straight pipe system [J]. Journal of Vibration Engineering, 2000, 13(3): 455-461.

3. Li Chenyang, Li Weijia, Li Tiecheng. Numerical analysis of sound field of hydraulic pipeline considering fluid- structure interaction [J]. Ship Science and Technology, 2011, 33(4): 25-29.

4. Chi Ting. Hydraulic calculation of steam pipeline and study on flow field characteristics of valve 
transient closing [D]. Harbin Engineering University, 2015.3.

5. Go Lufeng, Zheng Haicun. Hydraulic and thermal coupling for steam pipe network [J]. China Science Paper, 2013, 08:812-815.

6. Wng Hai. Analysis of multi-sources looped-pipe network based on object-oriented methodology [N]. Journal of Zhejiang University (Engineering science), 2012, 46 (10).

7. Yang Xueying. Flow analysis and optimization design of typical pipeline accessories [D]. Harbin Institute of Technology, 2012.7.

8. Wang Zeshen. The experiment and numerical simulation of fluid solid coupling vibration of water conveyance pipeline [D]. Harbin Institute of Technology, 2016.6.

9. Wang Daobin, Zhao Wenli, etc. Flow resistance characteristic analysis and structure optimization of brake pipeline based on FLUENT [J]. Mechanical and electrical engineering, 2012, 29(10): 1172-1174.

10. Liu Gongmin, Chen Hao, Li Shuaijun. Influence of steam parameters on dynamic characteristics of pipeline system [J]. Chinese Journal of Solid Mechanics, 2012, 33(2): 168-175.

11. Liu Zhongzu, Sun Yudong, Wu Yousheng. Research status and Prospect of fluid structure coupling vibration and sound propagation in pipelines [J]. Journal of Ship Mechanics, 2001, 5(2): 82-90 .

12. Wei Fayuan, Huang Yuying, Ren Zhiliang, etc. Transfer matrix method for analysis of critical velocity of curved pipe [J]. Chinese Journal of Solid Mechanics, 2000, 21(01): 33-39. 